\title{
Solving Some Differential Equations Arising in Electric Engineering Using Hermite Polynomials
}

\author{
G. Singh*, I. Singh \\ Department of Physical Sciences, Sant Baba Bhag Singh University, Jalandhar-144030, Punjab, India
}

Received 29 February 2020, accepted in final revised form 29 May 2020

\begin{abstract}
In this paper, a collocation method based on Hermite polynomials is presented for the numerical solution of the electric circuit equations arising in many branches of sciences and engineering. By using collocation points and Hermite polynomials, electric circuit equations are transformed into a system of linear algebraic equations with unknown Hermite coefficients. These unknown Hermite coefficients have been computed by solving such algebraic equations. To illustrate the accuracy of the proposed method some numerical examples are presented.
\end{abstract}

Keywords: Hermite polynomials; Applications; Numerical examples.

(C) 2020 JSR Publications. ISSN: 2070-0237 (Print); 2070-0245 (Online). All rights reserved. doi: http://dx.doi.org/10.3329/jsr.v12i4.45686

J. Sci. Res. 12 (4), 517-523 (2020)

\section{Introduction}

Differential equations arising in various engineering and science applications can be linear as well as nonlinear. The linear differential equations with constant coefficients have their significant applications in the study of electrical, mechanical and other linear systems. In fact such equations play a dominant role in unifying the theory of electrical and mechanical oscillatory systems. Numerical solution of series L-C-R is very useful in several branches of science and engineering. The collocation method has become very popular to solve differential equations. This method can reduce the complexity of solving the ordinary differential equations for epidemic models with high dimensions and it is very useful in finding the accurate solution to differential equations. In this research paper, Hermite polynomials, a class of the orthogonal polynomials that are orthogonal on $(-\infty, \infty)$, are used. Hermite polynomials have many advantages over other orthogonal polynomials. Hermite collocation method (HCM) has been used to solve linear and nonlinear ordinary differential equations with special initial conditions. The most important advantage of the proposed method is that it transforms any ordinary differential equations to a system of algebraic equations which can be solved easily.

${ }^{*}$ Corresponding author: gurpreet20794@gmail.com 
Gulsu et al. [1] proposed a collocation method based on Hermite polynomials for solving $m^{\text {th }}$ order linear differential-difference equations with variable coefficients under mixed conditions. In this method a truncated Hermite expansion of the function has been used in the differential-difference equations. Ayaz [2] proposed a numerical scheme based on an adaptation of the Taylor's collocation method in terms of truncated Hermite series to approximate the solution of the higher order fractional differential equations with multi terms. This method is very useful tool for solving fractional differential equations with variable coefficients. In Ibis and Bayramreport [3], a collocation method based on Hermite polynomials has been presented for solving the neutral functional-differential equations with proportional delays. Pirim et al. [4] proposed a numerical method for solving higher order linear Fredholmintegro-differential equations with variable coefficients. The proposed method converts the equation and its conditions to matrix equations, which correspond to a system of linear algebraic equations with unknown Hermite coefficients. The Hermite coefficients are obtained by solving matrix equation. Ayaz [5] presented an approximation method for solving a system of fractional order differential equations with variable coefficients by converting the mentioned system into a matrix equation which corresponds to a system of linear equations with unknown Hermite polynomial coefficients. In a study by Berwal et al. [6], numerical solution of series L-C$\mathrm{R}$ equations have been presented by using Haar wavelet method. Sofiand Singh [7] proposed a numerical scheme for solving L-C-R equations by using Laplace transform method. In a study by Secer et al. [8], Hermite collocation method has been used for solving the problem of the spread of a non-fatal disease in a population. Study of differential equations arising from the generating functions of Hermit Kampéde Fériet polynomials has been presented by Ryoo [9]. Hwang and Ryoo [10] investigate the structure and symmetry of zeros of the two variable degenerate Hermite equations. Singh [11] presented two numerical techniques such as Adomian decomposition and Haar wavelet methods for solving some oscillatory problems arising in several applications of science and engineering. Numerically investigation of the non-Newtonian viscoelastic Oldroyd-B fluid flows in two-dimensional rectangular domain has been discussed by Helal [12]. The numerical results of the nonlinear system of partial differential equations are obtained by decoupling the system into Navier-Stokes system and tensorial transport equation. Computational Fluid Dynamics (CFD) simulations are done by using the finite element method.

Hermite polynomials constitute a sequence of orthogonal polynomials that play a significant role in pure and computational mathematics, as well as in many branches of numerical analysis. These polynomials play an important role in spectral methods and these methods have been successfully applied in the approximation of partial, differential and integral equations. Most effectively used spectral methods are the Galerkin and collocation methods and the utility of these methods is based on the fact that if the solution sought is smooth, usually only a few terms in an expansion of global basis functions are needed to represent it to high accuracy. The most important advantage of 
proposed method is that it converts the given differential equation into a system of algebraic equations, which can be easily solved with the help of any classical method.

The main objective of this research is to solve some mathematical models arising in electric engineering by expanding the highest derivative appearing in differential equation into a series of Hermite polynomials. The other derivatives are obtained by using integration. To evaluate the numerical solution of differential equation, substituting all these derivatives into the given differential equation and discretized it with collocation points.

\section{Formulation of Different Differential Equation}

R-L series circuit: Consider a circuit containing resistance $(R)$ and inductance $(L)$ in series with a voltage source (battery) (V). Let $I$ be the current flowing in the circuit at any time $T$. Then by Kirchhoff's first law, the sum of voltage drops across $R$ and $L$ is equal to $V$. Therefore, we obtain,

$$
\frac{d I}{d T}+\frac{R}{L} I=\frac{V}{L}
$$

with initial condition $I(0)=0$. The exact solution of $(1)$ is,

$$
I=\frac{V}{R}\left(1-e^{-\frac{R T}{L}}\right)
$$

L-C-R series circuit: Consider an electrical circuit containing an inductance $L$, capacitance $C$ and resistance $R$. Consider the discharge of a condenser $C$ through an inductance $L$ and the resistance $R$. Since the voltage drop across $L, C$ and $R$ respectively

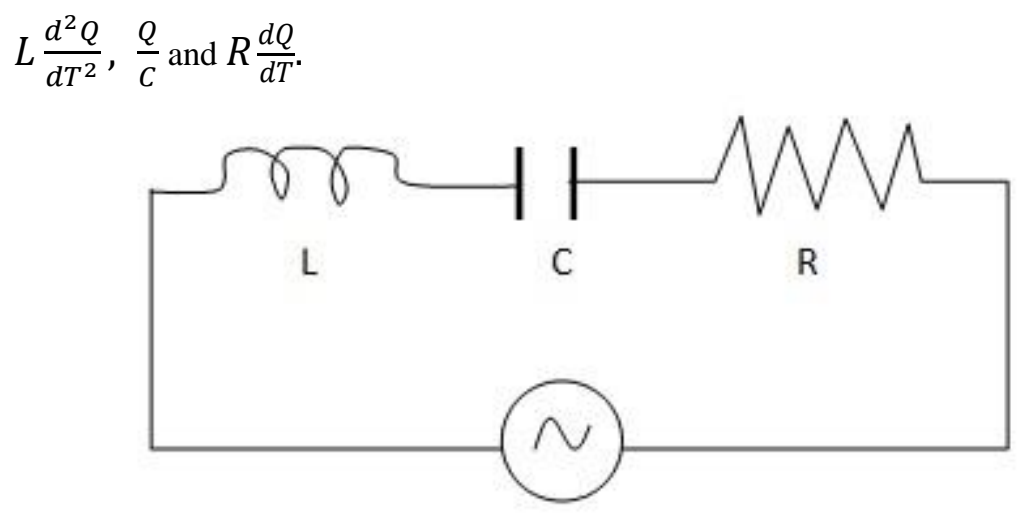

Fig. 1. L-C-R circuit.

A method based on Hermite polynomials can be applied to compute the charge on the capacitors and currents as function of time. A simple electrical circuit consists of the following elements connected in series (as shown in Fig. 1):

(i) a battery which supplied an electromotive force (E.M.F). 
(ii) an inductor that has inductance $\mathrm{L}$.

(iii) a resistance $\mathrm{R}$ and

(iv) a capacitor that has capacitance $\mathrm{C}$.

The differential equation of the above diagram is

$$
L \frac{d^{2} Q}{d T^{2}}+R \frac{d Q}{d T}+\frac{Q}{C}=E, \quad Q(0)=0, Q^{\prime}(0)=0
$$

where $Q$ is charge, $L$ is inductor, $R$ is resistor, $C$ is capacitor and $E$ is electromotive force. In this research paper, we are using the following special functions to obtain the numerical solution of these differential equations.

\section{Hermite's Polynomials}

These are the solutions of Hermite's differential equation

$$
y^{\prime \prime}-2 x y^{\prime}+2 n y=0
$$

These polynomials $H_{n}(x)$, are given by

$$
H_{n}(x)=(-1)^{n} e^{x^{2}} \frac{d^{n}}{d x^{n}}\left(e^{-x^{2}}\right)
$$

In particular,

$$
\begin{gathered}
H_{0}(x)=1, \\
H_{1}(x)=2 x, \\
H_{2}(x)=4 x^{2}-2, \\
H_{3}(x)=8 x^{3}-12 x, \\
H_{4}(x)=16 x^{4}-48 x^{2}+12, \\
H_{5}(x)=32 x^{5}-160 x^{3}+120 x, \\
H_{6}(x)=64 x^{6}-480 x^{4}+720 x^{2}-120, \\
H_{7}(x)=128 x^{7}-1344 x^{5}+3360 x^{3}-1680 x
\end{gathered}
$$

and so on. The orthogonal property of these polynomials is

$$
\int_{-\infty}^{\infty} e^{-x^{2}} H_{m}(x) H_{n}(x) d x= \begin{cases}0, & m \neq n \\ 2^{n} n ! \sqrt{\pi}, & m=n\end{cases}
$$

\section{Applications}

In this section, we present some numerical experiments to illustrate the accuracy of the proposed method.

Example 1, (R- L series circuit): Letting $R=0.2 \mathrm{Ohms}, L=1 \mathrm{H}$ and $V=110 \mathrm{~V}$. The differential equation (1) becomes

$$
I^{\prime}+0.2 I=110
$$

Consider the approximation 


$$
I^{\prime}(T)=\sum_{i=0}^{N} a_{i} H e_{i}(T)
$$

Integrating with respect to $T$, from 0 to $T$, we obtain

$$
I(T)=I(0)+\sum_{i=0}^{N} a_{i} \int_{0}^{T} H e_{i}(T) d T
$$

Substituting the value of $I, I^{\prime}$ in (3), we obtain

$$
\sum_{i=0}^{N} a_{i}\left[H e_{i}(T)+0.2 \int_{0}^{T} H e_{i}(T) d T\right]=110
$$

From here, we obtain constant coefficients. The numerical solution of (3) is obtained by substituting the value of coefficients into (5).

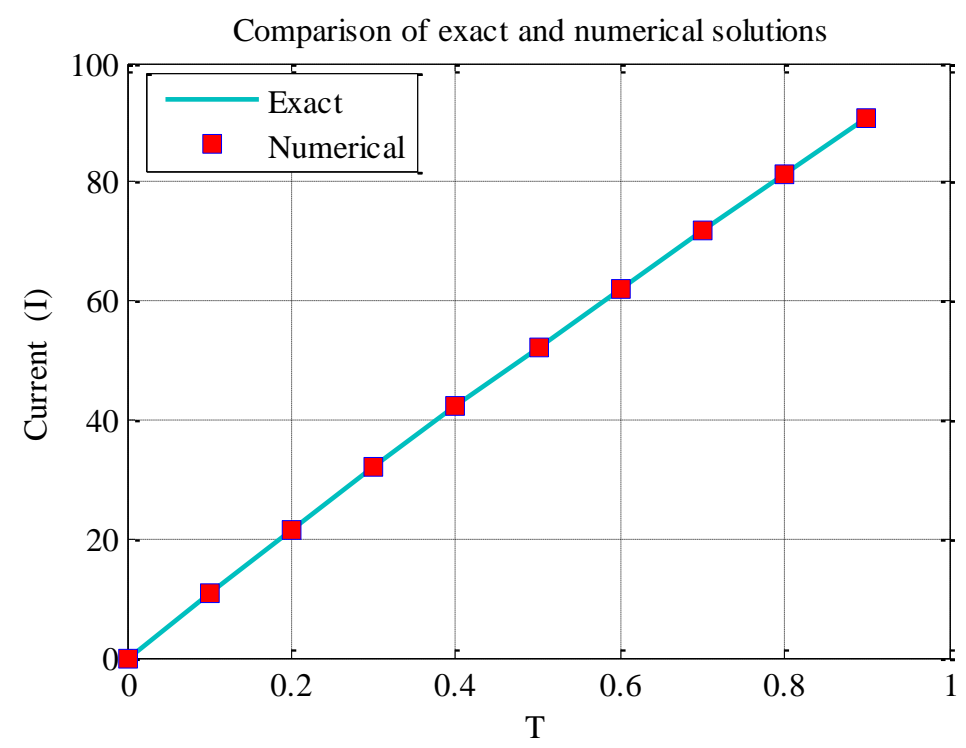

Fig. 2. Comparison of exact and numerical solutions of Example 1.

Example 2, (L-C-R series circuit): If an alternating E.M.F.Esin $(\omega T)$ is applied to an inductance $\mathrm{L}$ and a capacitance $\mathrm{C}$ in series, then the differential equation will be (where $\mathrm{R}$ $=0$ )

$$
L \frac{d^{2} Q}{d T^{2}}+\frac{Q}{C}=E \sin \omega T, \quad Q(0)=0, Q^{\prime}(0)=0
$$

The complete solution of the differential equation is

$$
Q(T)=C_{1} \cos \left(\frac{T}{\sqrt{L C}}\right)+C_{2} \sin \left(\frac{T}{\sqrt{L C}}\right)+\frac{E C}{\left(1-L C \omega^{2}\right)} \sin (\omega T)
$$

At $Q(0)=0$ and $Q^{\prime}(0)=0$, we have,

$$
C_{1}=0
$$




$$
C_{2}=-\frac{E C \omega}{\left(1-L C \omega^{2}\right)}
$$

Therefore, the exact solution of equation is

$$
Q(T)=-\frac{E C \omega}{\left(1-L C \omega^{2}\right)} \sqrt{L C} \sin \left(\frac{T}{\sqrt{L C}}\right)+\frac{E C}{\left(1-L C \omega^{2}\right)} \sin (\omega T)
$$

Consider the approximation

$$
Q^{\prime \prime}(T)=\sum_{i=0}^{N} a_{i} H e_{i}(T)
$$

Integrating with respect to $T$, from 0 to $T$, we get

$$
Q^{\prime}(T)=Q^{\prime}(0)+\sum_{i=0}^{N} a_{i} \int_{0}^{T} H e_{i}(T) d T
$$

Again, integrating with respect to $T$, from 0 to $\mathrm{T}$, we get

$$
Q(T)=Q(0)+T Q^{\prime}(0)+\sum_{i=0}^{N} a_{i} \int_{0}^{T} \int_{0}^{T} H e_{i}(T) d T d T
$$

Substituting these values in (6), we get

$$
\begin{aligned}
& L\left\{\sum_{i=0}^{N} a_{i} H e_{i}(T)\right\}+\frac{1}{C}\left\{Q(0)+T Q^{\prime}(0)+\sum_{i=0}^{N} a_{i} \int_{0}^{T} \int_{0}^{T} H e_{i}(T) d T d T\right\}=E \sin \omega T \\
& \left\{\sum_{i=0}^{N} a_{i}\left[L\left[H e_{i}(T)\right]+\frac{1}{C} \int_{0}^{T} \int_{0}^{T} H e_{i}(T) d T d T\right]\right\}=E \sin \omega T-\frac{1}{C}\left\{Q(0)+T Q^{\prime}(0)\right\}
\end{aligned}
$$

From here, coefficients $a_{0}, a_{1}, a_{2}, \ldots \ldots \ldots, a_{n}$ are obtained. The numerical solution is obtained by substituting the values of these coefficients into (7).

Case I: If $E=2 v, w=2$ radian, $C=2 \mu F$ and $L=1 H$.

Table 1. Comparison of exact and numerical solutions of Example 2 for Case I.

\begin{tabular}{cccc}
\hline $\mathrm{T}$ & Exact solution & Numerical solution & Absolute error \\
\hline 0.1 & 0.0000007833 & 0.0000007947 & $1.1369 \mathrm{e}-08$ \\
0.2 & 0.0000015582 & 0.0000015576 & $5.6993 \mathrm{e}-10$ \\
0.3 & 0.0000022698 & 0.0000022585 & $1.1279 \mathrm{e}-08$ \\
0.4 & 0.0000028683 & 0.0000028694 & $1.1228 \mathrm{e}-09$ \\
0.5 & 0.0000033546 & 0.0000033659 & $1.1228 \mathrm{e}-08$ \\
0.6 & 0.0000037298 & 0.0000037281 & $1.6779 \mathrm{e}-09$ \\
0.7 & 0.0000039529 & 0.0000039418 & $1.1144 \mathrm{e}-08$ \\
0.8 & 0.0000039960 & 0.0000039983 & $2.2420 \mathrm{e}-09$ \\
0.9 & 0.0000038843 & 0.0000038953 & $1.0971 \mathrm{e}-08$ \\
1.0 & 0.0000036400 & 0.0000036381 & $1.8507 \mathrm{e}-09$ \\
\hline
\end{tabular}


Fig. 2 shows the comparison of exact and numerical solutions of Example 1. Table 1 shows the comparison of exact and numerical solutions of Example 2 (Case 1). Table 2 shows the comparison of exact and numerical solutions of Example 2 (Case II).

Case II: If $E=4 v, w=1$ radian, $C=0.3 \mu F$ and $L=2 H$.

Table 2. Comparison of exact and numerical solutions of Example 2 for Case II.

\begin{tabular}{cccc}
\hline $\mathrm{T}$ & Exact solution & Numerical solution & Absolute error \\
\hline 0.1 & 0.0000001200 & 0.0000001198 & $2.6685 \mathrm{e}-10$ \\
0.2 & 0.0000002378 & 0.0000002384 & $5.1543 \mathrm{e}-10$ \\
0.3 & 0.0000003553 & 0.0000003546 & $7.1777 \mathrm{e}-10$ \\
0.4 & 0.0000004664 & 0.0000004673 & $8.5816 \mathrm{e}-10$ \\
0.5 & 0.0000005762 & 0.0000005753 & $9.2494 \mathrm{e}-10$ \\
0.6 & 0.0000006766 & 0.0000006775 & $9.1197 \mathrm{e}-10$ \\
0.7 & 0.0000007738 & 0.0000007730 & $8.2115 \mathrm{e}-10$ \\
0.8 & 0.0000008601 & 0.0000008608 & $6.5949 \mathrm{e}-10$ \\
0.9 & 0.0000009404 & 0.0000009399 & $4.4371 \mathrm{e}-10$ \\
1.0 & 0.0000010095 & 0.0000010098 & $2.2694 \mathrm{e}-10$ \\
\hline
\end{tabular}

\section{Conclusion}

The main goal of this paper is to demonstrate the method based on Hermite polynomials for solving series R-L and L-C-R equations. The algorithm and procedure have been applied by using Hermite polynomials for solving ODE's. The result is compared with the exact solution. It is worth mentioning that Hermite polynomials solution provides excellent result even for small values of N. From the above numerical data, we conclude that Hermite polynomials are working as accurate tool for solving ordinary differential equations.

\section{References}

1. M. Gulsu, H. Yalman, Y. Ozturk, and M. Sezer, Applicat. Appl. Math.: An Int. J. 6, 1856 (2011).

2. N. A. Pirim and F. Ayaz, An Int. J. Optimizat. Control: Theories Applcat. 8, 228 (2018).

3. B. Ibis and M. Bayram, Commun. Math. Model. Applicat. 1, 22 (2016).

4. A. N. Pirim, N. Sahin and M. Sezer, Numer. Methods Partial Differential Equat. 27, 1707 (2011). https://doi.org/10.1002/num.20604

5. A. N. Pirim and F. Ayaz, Appl. Math. 7, 2307 (2016). https://doi.org/10.4236/am.2016.718182

6. N. Berwal, D. Panchal, and C. L. Parihar, Ital. J. Pure Appl. Math. 30, 157 (2013).

7. M. A. Sofi and I. Singh, Int. J. Adv. Sci. Res. Manag. 4, 89 (2019).

8. A. Secer, N. Ozdemir, and M. Bayram, Mathematics 6, 305 (2018). https://doi.org/10.3390/math6120305

9. C. S. Ryoo, Mathematics 7, 23 (2019). https://doi.org/10.3390/math7010023

10. K. W. Hwang and C. S. Ryoo, Mathematics 8, 228 (2020). https://doi.org/10.3390/math8020228

11. I. Haq and I. Singh, J. Sci. Res. 12, 289 (2020). https://doi.org/10.3329/jsr.v12i3.44287

12. K. M. Helal, J. Sci. Res. 12, 327 (2020). https://doi.org/10.3329/jsr.v12i3.44970 